\title{
Involvement of Inflammation in Venous Thromboembolic Disease: An Update in the Age of COVID-19
}

\author{
Peter Poredos, MD, $\mathrm{PhD}^{1}$ Pavel Poredos, MD, $\mathrm{PhD}^{2}$ \\ ${ }^{1}$ Department of Anesthesiology and Perioperative Intensive Care, \\ University Medical Centre Ljubljana, Ljubljana, Slovenia \\ 2 Department of Vascular Disease, University Medical Centre \\ Ljubljana, Ljubljana, Slovenia \\ Address for correspondence Peter Poredos, MD, PhD, University \\ Medical Centre Ljubljana, Department of Anesthesiology and \\ Perioperative Intensive Care, Zaloska 7, Ljubljana SI-1000, Slovenia \\ (e-mail: peter.poredos@kclj.si).
}

Semin Thromb Hemost 2022;48:93-99.

\begin{abstract}
Keywords

- inflammation

- venous thromboembolism

- risk factors

- COVID-19

- anti-inflammatory treatment

The inflammatory process is strongly involved in the pathophysiology of venous thromboembolism (VTE) and has a significant role in disease prediction. Inflammation most probably represents a common denominator through which classical and nonclassical risk factors stimulate thrombotic process. Inflammation of the venous wall promotes the release of tissue factor, inhibits the release of anticoagulant factors, and hampers endogenous fibrinolysis. Systemic inflammatory response also inhibits restoration of blood flow in the occluded vessel. Recent studies indicate that increased inflammatory response ("cytokine storm") is related to prothrombotic state and thromboembolic events in patients with coronavirus disease 2019 (COVID-19). The growing evidence of involvement of inflammation in the pathogenesis of VTE indicates the importance of anti-inflammatory treatment and prevention of VTE. While aspirin was shown to be effective in prevention of recurrent venous thrombosis after treatment with anticoagulant drugs, some other anti-inflammatory drugs like nonsteroidal anti-inflammatory agents may have prothrombotic effect, thus potentially increasing the risk of VTE. Recently, new specific anti-inflammatory drug inhibitors of inflammatory markers that have been shown to be involved in the pathogenesis of VTE are being searched. As thrombogenesis is based on activation of coagulation provoked by inflammation, then prevention and treatment of VTE should include both anticoagulant and anti-inflammatory agents. Combined treatment is related to increased risk of bleeding complications, therefore subtherapeutic doses of both drugs should be used to improve the efficacy of management of VTE without increasing the risk of bleeding.
\end{abstract}

Systemic inflammation represents a potent prothrombotic stimulus. It is well known that inflammation is involved in the pathogenesis of atherothrombosis and recently it has been shown that inflammation also represents one of the basic pathogenetic mechanisms of venous thromboembolism (VTE). Inflammation most probably represents a common denominator through which different risk factors damage vessel wall, evoke inflammation, and trigger thrombus formation. ${ }^{1,2}$

Not only mechanical factors (turbulent blood flow) but also biochemical factors, in combination with a procoagulant state, stimulate thrombus formation. Inflammation of the vessel wall, which is usually induced by vessel wall injury, activates the coagulation system through an increase of tissue
Issue Theme Maintaining Hemostasis and Preventing Thrombosis in COVID-19-Part III; Guest Editors: Emmanuel J. Favaloro, PhD, FFSc (RCPA) and Giuseppi Lippi, MD (c) 2021. Thieme. All rights reserved. Thieme Medical Publishers, Inc., 333 Seventh Avenue, 18th Floor, New York, NY 10001, USA
DOI https://doi.org/ 10.1055/s-0041-1732372. ISSN 0094-6176. 
Table 1 Procoagulant effects of inflammation

\begin{tabular}{|c|c|}
\hline Mechanisms & Procoagulant effects \\
\hline \multicolumn{2}{|l|}{ Activation of inflammatory cells } \\
\hline Platelets & $\begin{array}{l}\text { Release of polyphosphate from dense granules: } \\
\text { - Activation of FV, FXII. } \\
\text { - Inhibition of TFPI activity. } \\
\text { - Activation of TAFI. }\end{array}$ \\
\hline Neutrophils & $\begin{array}{l}\text { NETS formation: } \\
\text { - Activation of FXI, FXII. } \\
\text { - Impairment of thrombomodulin-dependent protein C activation. } \\
\text { - Inhibition of TFPI. }\end{array}$ \\
\hline Activation of procoagulant factors & Direct activation of TF driven coagulation pathways \\
\hline Microparticles formation & $\begin{array}{l}\text { Release of PSGL-1: } \\
\text { - Activation of TF. }\end{array}$ \\
\hline Endothelial damage & Endothelial dysfunction and loss of antithrombotic properties \\
\hline
\end{tabular}

Abbreviations: NET, neutrophil extracellular trap; PSGL-1, P-selectin glycoprotein ligand-1; TAFI, thrombin activatable fibrinolysis inhibitor; TF, tissue factor; TFPI, tissue factor pathway inhibitor.

factor expression. Inflammatory diseases, such as inflammatory bowel disease, some rheumatic diseases, and cancer lead to increased release of inflammatory cytokines that stimulate coagulation and platelet activation. Inflammation also damages the endothelium, which consequently loses its anticoagulant properties and triggers blood coagulation. ${ }^{3}$ Further, increased thromboembolic risk of coronavirus disease 2019 (COVID-19) is associated with inflammatory response when elevated levels of pro-inflammatory cytokines cause damage to endothelium and lead to prothrombotic endothelial dysfunction. 4

Therefore, systemic inflammation is a potent prothrombotic stimulus which upregulates procoagulant factors, downregulates natural anticoagulants, and inhibits fibrinolytic activity.

In patients with COVID-19, especially in the critical form of disease, an exaggerated inflammatory response defined as a "cytokine storm" has been reported. ${ }^{5}$ This resembles other hyperinflammation syndromes, falling into the broader definition of cytokine release syndrome.

\section{Interrelationship between Inflammation and Coagulation}

Thrombus formation is attributed to three main groups of factors, including alteration in blood flow, endothelial injury, and hypercoagulable state, which contribute to the wellknown Virchow's triade. ${ }^{6}$ Recently, a growing body of evidence suggests a role of inflammation as a major contributor to pathogenesis of VTE, ${ }^{7}$ by enhancing the hypercoagulable state and causing endothelial damage. The key event in the initiation of VTE is most probably venous wall inflammation. Thrombus formation starts with activation and damage of endothelial cells, platelets, and leukocytes which subsequently initiate inflammation and microparticle formation that trigger the coagulation system through the stimulation of tissue factor
(TF) release. Monocytic TF and activation of the intrinsic pathway with neutrophils promote thrombus formation. ${ }^{4}$

Neutrophils represent important but under-recognized players, not only in the immune system as launching the first line of defense against invading microorganisms, but also promoting coagulation. Activated neutrophils release their decondensed chromatin as a network of extracellular fibersneutrophil extracellular traps (NETs). ${ }^{8}$ NETs are composed of DNA and histones that exert antimicrobial properties. ${ }^{9}$ Further, NETs also stimulate the activation of coagulation (both extrinsic and intrinsic pathways) and platelet adhesion. Chromatin network activates coagulation also through binding of factor XII and XI, impairs thrombomodulin-dependent protein $\mathrm{C}$ activation, and inhibits TF pathway inhibitor. ${ }^{10}$

The link between coagulation and inflammation is also supported by polyphosphate, which is present in human platelet dense granules and released upon platelet activation. Polyphosphate stimulates coagulation by increasing activation of factor $\mathrm{V}$, decreasing TF pathway inhibitor (TFPI) activity, and delaying clot lysis by activation of thrombin activatable fibrinolysis inhibitor (TAFI) ${ }^{11}$ Inflammatory markers increase the number of microparticles through leukocyte activation and concentration of TF at particle surfaces. Microparticles bearing TF and P-selectin glycoprotein ligand-1 (PSGL-1) allow TF to express coagulant activity $^{12}$ (-Table 1, - Fig. 1). Recently, an association between VTE and several markers of inflammation such as C-reactive protein (CRP), interleukin-6 (IL-6), interleukin-8 (IL-8), and tumor necrosis factor $\alpha$ (TNF $\alpha$ ) has been demonstrated. ${ }^{2,13}$ These pro-inflammatory cytokines play an important role in pathogenesis of VTE by promoting a procoagulant state primarily by inducing TF expression. Several immune system components (cytokines, chemokines, and various leukocyte subtypes) are involved in the inflammatory process of VTE. ${ }^{14}$ Additionally, other inflammatory mediators such as polyphosphates and bradykinin may directly activate TF driven coagulation pathways. ${ }^{15}$ 


\section{INFLAMMATION and VTE}

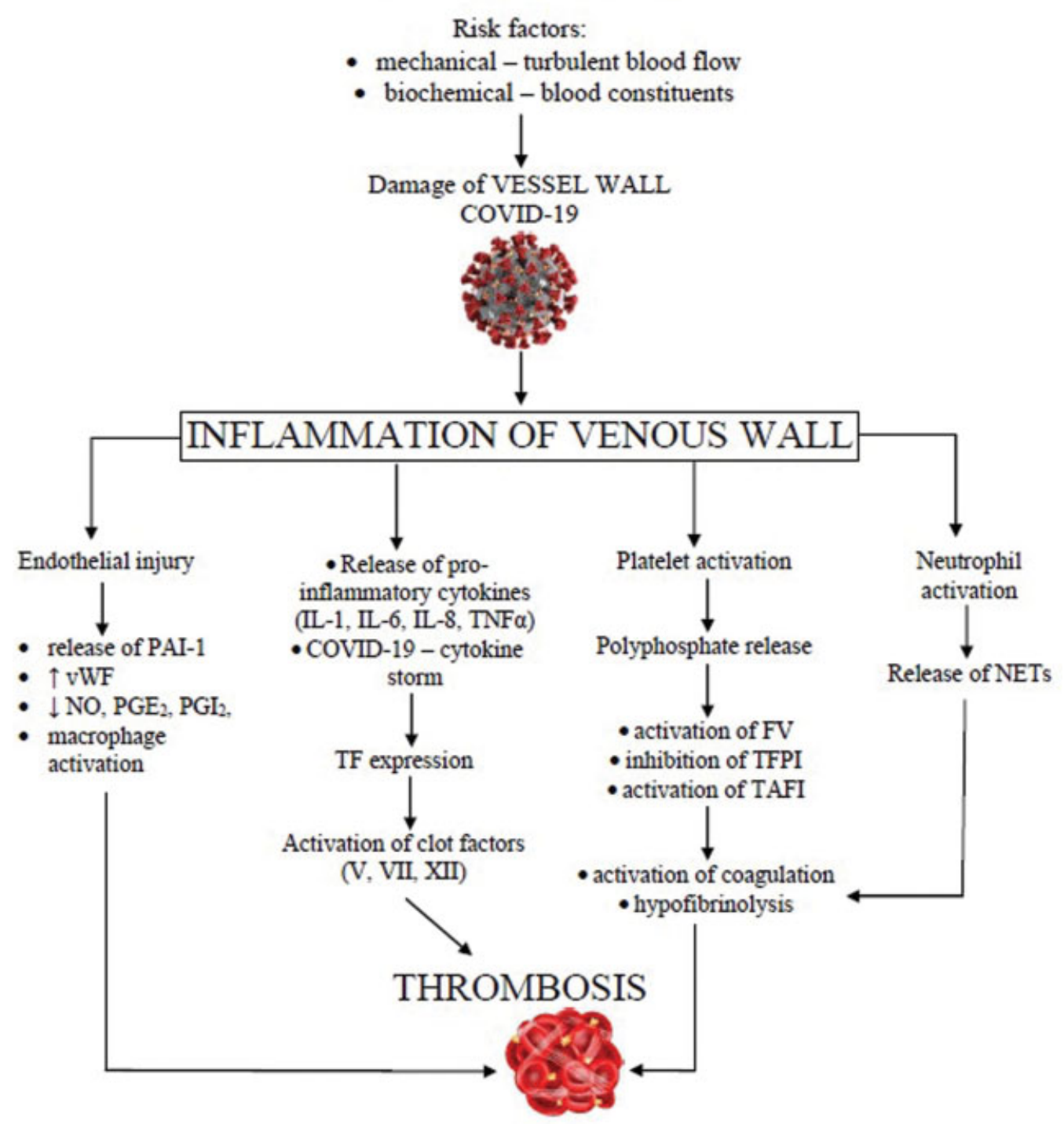

Fig. 1 Involvement of inflammation in venous thrombosis. IL-1, IL-6, IL-8, interleukins; TAFI, thrombin activatable fibrinolysis inhibitor; TFPI, tissue factor pathway inhibitor; TNF $\alpha$, tumor necrosis factor $\alpha$; VWF, von Willebrand factor; VTE, venous thromboembolic disease.

The relationship between inflammation and VTE is supported by increased levels of markers of inflammation such as CRP, IL-6, IL-8, and TNF- $\alpha{ }^{16,17}$ One study showed that patients with idiopathic venous thrombosis in stable phase (3-4 months after acute disease) have increased levels of circulating markers of inflammation (CRP, IL-6, IL-8); also, anti-inflammatory interleukin-10 (IL-10) was significantly decreased. This would indicate that patients still had increased systemic inflammatory responses after the acute phase of disease. Increased inflammatory markers were related to markers of endothelial dysfunction. ${ }^{18}$ To elucidate the dilemma if increased levels of inflammatory markers in patients with VTE are active players or simple bystanders, this group of patients was followed up for 4 years. In patients without a complete recanalization of previously occluded veins, inflammatory markers (CRP, IL-8) were increased, and anti-inflammatory
IL-10 was again decreased. This indicates that a systemic inflammatory response is most probably not the consequence of the disease but is actively involved in its pathogenesis. ${ }^{19}$ These results confirmed that the inflammatory markers, particularly pro-inflammatory cytokines, are involved in the pathogenesis of VTE by promoting a procoagulant state. ${ }^{14}$

Therefore, there is an extensive crosstalk between coagulation and inflammation, whereby activation of one system may amplify activation of the other. ${ }^{20}$ Vice-versa coagulation processes stimulate inflammatory responses. TF triggers not only the generation of active coagulation factors, but also induces protease-activated receptor-mediated signaling, which stimulates the release of more inflammatory cytokines, activates leukocytes and vascular cell adhesion molecules and suppresses vasoprotective molecules, such as thrombomodulin. ${ }^{21}$ Therefore, activated coagulation proteases stimulate specific 
receptors on inflammatory cells and endothelial cells and thereby modulate the inflammatory response. ${ }^{22}$

\section{The Relationship between Inflammation, Coagulation Factor XII and Thrombosis}

The crosstalk between coagulation and inflammation is further indicated by the activity of factor XII driven contact pathway, which is involved in coagulation and inflammation via the intrinsic pathway of coagulation and the bradykinin-producing kallikrein-kinin system. ${ }^{23}$ Coagulation factor XII is plasma protein, and the zymogen form of factor XIla, an enzyme of the serin protease class. In vitro, FXII is activated to FXIIa after binding to negatively charged surfaces, such as glass. In vivo, FXII is activated by contact with polyanions. Contact to polyphosphates released by activated platelets activates FXII and this then promotes fibrin formation via the intrinsic pathway of coagulation. ${ }^{24}$ FIla cleaves plasma kallikrein and generates active kallikrein which in turn reciprocally activates additional FXII. Besides activation of the intrinsic pathway of coagulation, this process also liberates the inflammatory mediator bradykinin by kallikrein-mediated cleavage of high molecular weight kininogen. ${ }^{24}$ Therefore, excessive FXII activity is associated with a life-threatening inflammatory disorder called hereditary angioedema. The contact system and its activator polyphosphate have been recognized as important contributors to thromboembolic and inflammatory disorders. On the other hand, balanced and targeting FIla and polyphosphate interferes with the polyphosphate/FXII axis, providing safe thromboprotection and anti-inflammatory activity. Therefore, polyphosphatase may serve as a potent anti-inflammatory agent in reducing FIIa-driven complement and bradykinin effects. ${ }^{25}$

\section{Inflammation and Fibrinolysis}

Inflammation is involved in initiation and resolution of venous thrombi. Neutrophils in venous thrombus play a critical role during the early phase of venous thrombus resolution and collagen lysis. ${ }^{26,27}$ In addition, neutrophils facilitate the recruitment of monocytes into the thrombus, where they produce various chemokines and matrix-degrading proteases and stimulate thrombus resolution. In contrast, some inflammatory markers, particularly interleukins (e.g., IL-6) inhibit fibrinolysis and can be involved in fibrotic reorganization of thrombus. In the study of Nosaka and coworkers, it was shown that neutralization of IL- 6 with specific antibodies accelerated thrombus resolution and decreased vein wall fibrosis. ${ }^{28}$ One of our studies, which included patients with superficial venous thrombosis where the recanalization rate and extent of thrombus was followed up to 1 year, showed that the recanalization rate is negatively associated with level of inflammatory biomarkers. Patients with a lower recanalization rate had increased levels of CRP, IL-6, and TNF- $\alpha .{ }^{29}$ In contrast, administration of exogenous TNF- $\alpha$ accelerated thrombus resolution in mice and TNF- $\alpha$ antibody (TNF- $\alpha$ inhibitor) retarded venous thrombus resolution. ${ }^{28}$
In tissue injury and disease, the proteases that activate factor X and VII (to FXa, FVIIa), thrombin, plasmin and tissue plasminogen activator (t-PA) not only participate in coagulation or fibrinolysis but also mediate inflammation and tissue remodeling. ${ }^{30}$ TNF $\alpha$ and IL-1 decrease t-PA levels in human umbilical vein endothelial cells. ${ }^{31}$ Recent evidence also suggests that plasminogen-activator inhibitor-1 (PAI-1) is tightly associated with inflammation and that PAI-1 levels are locally enhanced in inflammatory sites of vessel wall. ${ }^{32}$

\section{COVID-19, VTE, and Inflammation}

Recent findings indicate that there is increased risk of VTE associated with COVID-19. COVID-19-related VTE is associated with higher risk of morbidity and mortality. ${ }^{33}$ Meta-analysis has shown that thromboembolism significantly increases the odds of mortality of COVID-19, which can be as high as $74 \%^{33}$ Risk factors of thromboembolic complications in patients with the COVID-19 have not been completely elucidated. A metaanalysis of studies that reported thromboembolisms in patients with COVID-19 showed that increasing age was associated with a higher prevalence of VTE and pulmonary embolism, while increased body-mass index was associated only with increased prevalence of pulmonary embolism. ${ }^{34}$ The mortality of patients with COVID-19 is related to the seriousness of the disease. Data from the RIETE registry (Registro Informatizado della Enfermedad ThromboEmbolica) showed that 10-day mortality among patients at a hospital ward was 9.1\% whereas it was $19.0 \%$ among those treated in intensive care units. ${ }^{35}$ To reduce thromboembolism occurrence, thromboprophylaxis in patients hospitalized because of COVID-19 is now well established. The studies also showed that postdischarge thromboprophylaxis is beneficial, particularly in higher risk patients, and revealed lower incidence of VTE. ${ }^{36}$

There exist different mechanisms through which COVID-19 contributes to elevated thromboembolism risk. Most probably, systemic inflammatory processes represent the basic mechanism of increased prothrombotic state. Abnormally elevated levels of pro-inflammatory cytokines (cytokine storm) have been found in COVID-19 patients. ${ }^{37}$ Several studies suggested that cytokines levels, particularly IL-1, TNF- $\alpha$ and IL-6 correlated directly with lung injury and multiple organ failure. ${ }^{38}$ The increased systemic inflammation causes endothelial injury and leads to pro-thrombotic endothelial dysfunction. ${ }^{39}$ One recent autopsy study found that almost no organ in the body is saved from thrombosis. ${ }^{40}$ Significant macrovascular and microvascular thrombosis was found in multiple organs. As the disease progresses, widespread thrombosis and multiorgan dysfunction syndrome appear. ${ }^{41}$

The rates of VTE in COVID-19 patients are higher than in other viral pandemics experienced in the past, ${ }^{42}$ and there is a correlation between disease severity and the risk of thromboembolism among SARS-CoV-2 infected individuals. Higher infectious dose is followed by active and prolonged viral replication in pneumocytes, macrophages and other immune cells. ${ }^{43}$

Activation of coagulation during virus infections could be the consequence of increased platelet adhesion and aggregation, and high release of inflammatory cytokines 
("cytokine storm"). ${ }^{44}$ The enhanced cytokine production also stimulates procoagulant reactions, with increased TF expression, a major initiator of the coagulation. Thrombin, which leads to fibrin formation, enhances platelet activation and alters fibrinolysis, both of which are also major mediators of inflammatory response. As inflammation results in further TF expression, thrombin generation represents initiation of a vicious cycle, which promotes the procoagulant state..$^{23,45}$ Neutrophil extracellular traps and damage-associated molecular patterns may also be involved in the procoagulant profile in patients with COVID-19. ${ }^{46}$

\section{Inflammation Therapeutic Target in the Management of VTE}

Anticoagulation therapy represents an effective option in preventing VTE and the propagation of VTE events. However, not only prevention of thrombus propagation but faster resolution of the thrombus is the key for improvement of disease prognosis. As inflammation represents one of the basic pathogenetic mechanism of VTE and is involved in thrombolysis and elimination of thrombus, it is expected that inhibition of inflammation, together with anticoagulation, may improve the efficacy of prevention of thromboembolic events and induce recanalization of thrombotic occlusions of veins. ${ }^{47}$ One of the first drugs which was used for the prevention of VTE was aspirin, which also has anti-inflammatory properties. ${ }^{48}$ It was shown that in higherrisk medical or surgical patients, aspirin reduces the incidence of VTE. However, the evidence of efficacy of aspirin in the primary prevention of VTE was too weak for general recommendation to use aspirin in the primary prevention of VTE. ${ }^{49}$

However, recent studies and meta-analysis have shown that aspirin is effective in the prevention of recurrent VTE as extended treatment of patients who completed initial anticoagulant treatment. The INSPIRE collaboration study showed that aspirin, after anticoagulant treatment of patients with the first unprovoked VTE, reduces the overall risk of VTE reoccurrence by more than one-third, without significantly increasing the risk of bleeding. ${ }^{50}$ In another study where aspirin was compared to placebo, aspirin did not significantly reduce the rate of recurrent VTE, but it significantly reduced the rate of major vascular events. This result shows that in patients, aspirin may have some antithrombotic therapeutic benefits ${ }^{51}$ after initial anticoagulant therapy with unprovoked venous thrombosis, which is presumably based on its antiinflammatory activity.

Although the studies highlight the importance of inflammation in pathogenesis of VTE and anti-inflammatory treatment for prevention and management of patients with thrombotic diseases, the studies indicated that the effects of anti-inflammatory drugs on coagulation and thrombus formation differ. It was shown that nonsteroidal anti-inflammatory drugs (NSAID) or cyclooxygenase-2-selective (COX-2) inhibitors increase the risk of VTE. ${ }^{52} \mathrm{~A}$ recent meta-analysis indicated that NSAID users have 1.8-times higher risk for VTE. ${ }^{53}$ As a relationship between some inflammatory markers (interleukins, selectins) and VTE was shown, the interest for the search of new anti-inflammatory drugs (which are also inhibitors of inflammatory markers) is growing. P-selectin, which is involved in the pathophysiology of VTE, has stimulated researchers to evaluate the ways of its inhibition. ${ }^{54}$ It was shown that P-selectin inhibition promotes thrombus resolution and prevents vein wall fibrosis. In a model of murine thrombosis induction, Pselectin inhibition with its inhibitor has been shown to decrease inflammation and reduce the risk for VTE. ${ }^{11}$ Interleukin and TNF- $\alpha$ inhibitors have shown effectiveness in prevention of atherosclerotic cardiovascular events. ${ }^{55}$ Therefore, they are being investigated as potential drugs also for the prevention and treatment of VTE.

In COVID-19 patients beside anticoagulant drugs also antiplatelet agents such as aspirin, ticagrelor, and dipyridamole drugs for the prevention of thrombosis are used and preliminary results showed that they could be effective. ${ }^{56}$

Further, drugs with a pleiotropic anti-inflammatory effect like statins are reported to reduce the occurrence of VTE. ${ }^{57}$ Therefore, current evidence would support a favorable effect of statins as adjuvant therapy in patients with COVID-19. ${ }^{58}$ Statins have shown anti-inflammatory effects in rodent models of thrombosis with the reduction of inflammatory biomarkers, including cytokines and P-selectins, neutrophil, and macrophage infiltrations within the thrombi and vessel wall. $^{59}$

Heparins also have reported to have anti-inflammatory properties and reduce different mediators of inflammation. ${ }^{60}$ Heparins reduced inflammation in relation to their ability to inhibit factor Xa and thrombin. ${ }^{61}$ Newer direct oral anticoagulants (DOACs) have also shown direct anti-inflammatory potential. ${ }^{62}$ It may explain their profibrinolytic potential and higher recanalization rates of VTE in DOAC anticoagulated patients compared to warfarin. ${ }^{63}$

\section{Conclusion}

There is an extensive crosstalk between coagulation and inflammation. Inflammation promotes prothrombotic state through TF expression, thrombin generation, inhibition of release of anticoagulant factors, and activation of inflammatory cells. Further, inflammation inhibits endogenic fibrinolysis and causes prothrombotic endothelial dysfunction. Vice-versa, activation of the coagulation system may importantly affect inflammatory response by different mechanisms. In patients with COVID-19, an intensive inflammatory response known as "cytokine storm" is most probably responsible for the hypercoagulable state leading to widespread thrombosis.

As inflammation represent one the basic pathogenetic mechanisms of VTE, it is expected that inhibition of inflammation together with anticoagulation may improve efficacy of prevention and treatment of thromboembolic events. Therefore, recent interest for searching new anti-inflammatory drugs, particularly inhibitors of inflammatory markers, is growing. 


\section{Conflict of Interest}

None declared.

\section{References}

1 Aksu K, Donmez A, Keser G. Inflammation-induced thrombosis: mechanisms, disease associations and management. Curr Pharm Des 2012;18(11):1478-1493

2 Poredos P, Jezovnik MK. The role of inflammation in venous thromboembolism and the link between arterial and venous thrombosis. Int Angiol 2007;26(04):306-311

3 Tieken C, Versteeg HH. Anticoagulants versus cancer. Thromb Res 2016;140(Suppl 1):S148-S153

4 Rodger M, Versteeg HH. An inflammatory fascination for thrombosis. Thromb Res 2016;144:224-225

5 Lippi G, Plebani M. Cytokine “storm”, cytokine “breeze”, or both in COVID-19? Clin Chem Lab Med 2021;59:637-639

6 Kyrle PA, Eichinger S. Deep vein thrombosis. Lancet 2005;365 (9465):1163-1174

7 Wakefield TW, Myers DD, Henke PK. Mechanisms of venous thrombosis and resolution. Arterioscler Thromb Vasc Biol 2008; 28(03):387-391

8 Laridan E, Martinod K, De Meyer SF. Neutrophil extracellular traps in arterial and venous thrombosis. Semin Thromb Hemost 2019; 45(01):86-93

9 Badimon L, Vilahur G. Neutrophil extracellular traps: a new source of tissue factor in atherothrombosis. Eur Heart J 2015;36 (22):1364-1366

10 Massberg S, Grahl L, von Bruehl ML, et al. Reciprocal coupling of coagulation and innate immunity via neutrophil serine proteases. Nat Med 2010;16(08):887-896

11 Branchford BR, Carpenter SL. The role of inflammation in venous thromboembolism. Front Pediatr 2018;6:142

12 Esmon CT. The interactions between inflammation and coagulation. Br J Haematol 2005;131(04):417-430

13 Gao Q, Zhang P, Wang W, et al. The correlation analysis of tumor necrosis factor-alpha-308G/A polymorphism and venous thromboembolism risk: a meta-analysis. Phlebology 2016;31(09): 625-631

14 Saghazadeh A, Hafizi S, Rezaei N. Inflammation in venous thromboembolism: cause or consequence? Int Immunopharmacol 2015;28(01):655-665

15 Long AT, Kenne E, Jung R, Fuchs TA, Renné T Contact system revisited: an interface between inflammation, coagulation, and innate immunity. J Thromb Haemost 2016;14(03):427-437

16 Folsom AR, Lutsey PL, Astor BC, Cushman M. C-reactive protein and venous thromboembolism. A prospective investigation in the ARIC cohort. Thromb Haemost 2009;102(04):615-619

17 Mahemuti A, Abudureheman K, Aihemaiti X, et al. Association of interleukin-6 and C-reactive protein genetic polymorphisms levels with venous thromboembolism. Chin Med J (Engl) 2012;125 (22):3997-4002

18 Jezovnik MK, Poredos P. Idiopathic venous thrombosis is related to systemic inflammatory response and to increased levels of circulating markers of endothelial dysfunction. Int Angiol 2010;29 (03):226-231

19 Jezovnik MK, Fareed J, Poredos P. Patients with a history of idiopathic deep venous thrombosis have long-term increased levels of inflammatory markers and markers of endothelial damage. Clin Appl Thromb Hemost 2017;23(02):124-131

20 Foley JH, Conway EM. Cross talk pathways between coagulation and inflammation. Circ Res 2016;118(09):1392-1408

21 Sower LE, Froelich CJ, Carney DH, Fenton JW II, Klimpel GR. Thrombin induces IL-6 production in fibroblasts and epithelial cells. Evidence for the involvement of the seven-transmembrane domain (STD) receptor for alpha-thrombin. J Immunol 1995;155 (02):895-901
22 O'Brien M. The reciprocal relationship between inflammation and coagulation. Top Companion Anim Med 2012;27(02):46-52

23 Maas C, Renné T Coagulation factor XII in thrombosis and inflammation. Blood 2018;131(17):1903-1909

24 Müller F, Mutch NJ, Schenk WA, et al. Platelet polyphosphates are proinflammatory and procoagulant mediators in vivo. Cell 2009; 139(06):1143-1156

25 Renné T, Schuh K, Müller-Esterl W. Local bradykinin formation is controlled by glycosaminoglycans. J Immunol 2005;175(05): 3377-3385

26 Ortega-Gómez A, Perretti M, Soehnlein O. Resolution of inflammation: an integrated view. EMBO Mol Med 2013;5(05):661-674

27 Mackman N. New insights into the mechanisms of venous thrombosis. J Clin Invest 2012;122(07):2331-2336

28 Nosaka M, Ishida Y, Kimura A, et al. Contribution of the TNF- $\alpha$ (Tumor Necrosis Factor- $\alpha$ )-TNF-Rp55 (tumor necrosis factor receptor p55) axis in the resolution of venous thrombus. Arterioscler Thromb Vasc Biol 2018;38(11):2638-2650

29 Poredoš P, Spirkoska A, Ježovnik MK. In patients with superficial vein thrombosis the inflammatory response is increased and related to the recanalization rate. Arch Med Sci 2019;15(02):393-401

30 Schuliga M. The inflammatory actions of coagulant and fibrinolytic proteases in disease. Mediators Inflamm 2015;2015:437695

31 Larsson P, Ulfhammer E, Karlsson L, Bokarewa M, Wåhlander K, Jern S. Effects of IL-1beta and IL-6 on tissue-type plasminogen activator expression in vascular endothelial cells. Thromb Res 2008;123(02):342-351

32 Lin H, Xu L, Yu S, Hong W, Huang M, Xu P. Therapeutics targeting the fibrinolytic system. Exp Mol Med 2020;52(03):367-379

33 Malas MB, Naazie IN, Elsayed N, Mathlouthi A, Marmor R, Clary B. Thromboembolism risk of COVID-19 is high and associated with a higher risk of mortality: a systematic review and meta-analysis. EClinicalMedicine 2020;29:100639

34 Di Minno A, Ambrosino P, Calcaterra I, Di Minno MND. COVID-19 and venous thromboembolism: a meta-analysis of literature studies. Semin Thromb Hemost 2020;46(07):763-771

35 Fernández-Capitán C, Barba R, Díaz-Pedroche MDC, et al. Presenting characteristics, treatment patterns, and outcomes among patients with venous thromboembolism during hospitalization for COVID-19. Semin Thromb Hemost 2021;47(04):351-361

36 Engelen MM, Vandenbriele C, Balthazar T, et al. Venous thromboembolism in patients discharged after COVID-19 hospitalization. Semin Thromb Hemost 2021;47(04):362-371

37 Huang C, Wang Y, Li X, et al. Clinical features of patients infected with 2019 novel coronavirus in Wuhan, China. Lancet 2020;395 (10223):497-506

38 Gao Y, Li T, Han M, et al. Diagnostic utility of clinical laboratory data determinations for patients with the severe COVID-19. J Med Virol 2020;92(07):791-796

39 Henry BM, Vikse J, Benoit S, Favaloro EJ, Lippi G. Hyperinflammation and derangement of renin-angiotensin-aldosterone system in COVID-19: a novel hypothesis for clinically suspected hypercoagulopathy and microvascular immunothrombosis. Clin Chim Acta 2020;507:167-173

40 Rapkiewicz AV, Mai X, Carsons SE, et al. Megakaryocytes and platelet-fibrin thrombi characterize multi-organ thrombosis at autopsy in COVID-19: a case series. EClinicalMedicine 2020; 24:100434

41 Lippi G, Sanchis-Gomar F, Favaloro EJ, Lavie CJ, Henry BM. Coronavirus disease 2019-associated coagulopathy. Mayo Clin Proc 2021;96(01):203-217

42 Bunce PE, High SM, Nadjafi M, Stanley K, Liles WC, Christian MD. Pandemic H1N1 influenza infection and vascular thrombosis. Clin Infect Dis 2011;52(02):e14-e17

43 Vadasz Z, Brenner B, Toubi E. Immune-mediated coagulopathy in COVID-19 infection. Semin Thromb Hemost 2020;46(07):838-840

44 Connors JM, Levy JH. COVID-19 and its implications for thrombosis and anticoagulation. Blood 2020;135(23):2033-2040 
45 Benati M, Salvagno GL, Nitto S, et al. Thrombin generation in patients with coronavirus disease 2019. Semin Thromb Hemost 2021;47(04):447-450

46 Zuo Y, Yalavarthi S, Shi H, et al. Neutrophil extracellular traps in COVID-19. JCI Insight 2020;5(11):e138999

47 Poredoš P, Poredoš P, Jezovnik MK. Factors influencing recanalization of thrombotic venous occlusions. Vasa 2020;49(01):17-22

48 Lippi G, Favaloro EJ. Venous and arterial thromboses: two sides of the same coin? Semin Thromb Hemost 2018;44(03):239-248

49 Becattini C, Agnelli G. Aspirin for prevention and treatment of venous thromboembolism. Blood Rev 2014;28(03):103-108

50 Simes J, Becattini C, Agnelli G, et al; INSPIRE Study Investigators (International Collaboration of Aspirin Trials for Recurrent Venous Thromboembolism) Aspirin for the prevention of recurrent venous thromboembolism: the INSPIRE collaboration. Circulation 2014;130(13):1062-1071

51 Brighton TA, Eikelboom JW, Mann K, et al; ASPIRE Investigators. Low-dose aspirin for preventing recurrent venous thromboembolism. N Engl J Med 2012;367(21):1979-1987

52 Schmidt M, Christiansen CF, Horváth-Puhó E, Glynn RJ, Rothman KJ, Sørensen HT. Non-steroidal anti-inflammatory drug use and risk of venous thromboembolism. J Thromb Haemost 2011;9(07): 1326-1333

53 Ungprasert P, Srivali N, Wijarnpreecha K, Charoenpong P, Knight EL. Non-steroidal anti-inflammatory drugs and risk of venous thromboembolism: a systematic review and meta-analysis. Rheumatology (Oxford) 2015;54(04):736-742

54 Diaz JA, Wrobleski SK, Alvarado CM, et al. P-selectin inhibition therapeutically promotes thrombus resolution and prevents vein wall fibrosis better than enoxaparin and an inhibitor to von Willebrand factor. Arterioscler Thromb Vasc Biol 2015;35(04):829-837
55 Kosmas CE, Silverio D, Sourlas A, Montan PD, Guzman E, Garcia MJ. Anti-inflammatory therapy for cardiovascular disease. Ann Transl Med 2019;7(07):147

56 Yuan S, Chen P, Li H, Chen C, Wang F, Wang DW. Mortality and prehospitalization use of low-dose aspirin in COVID-19 patients with coronary artery disease. J Cell Mol Med 2020

57 Glynn RJ, Danielson E, Fonseca FA, et al. A randomized trial of rosuvastatin in the prevention of venous thromboembolism. $\mathrm{N}$ Engl J Med 2009;360(18):1851-1861

58 Onorato D, Pucci M, Carpene G, Henry BM, Sanchis-Gomar F, Lippi G. Protective effects of statins administration in European and North American patients infected with COVID-19: a metaanalysis. Semin Thromb Hemost 2021;47(04):392-399

59 Feng Y, Lei B, Zhang F, Niu L, Zhang H, Zhang M. Anti-inflammatory effects of simvastatin during the resolution phase of experimentally formed venous thrombi. J Investig Med 2017;65(06): 999-1007

60 Poterucha TJ, Libby P, Goldhaber SZ. More than an anticoagulant: Do heparins have direct anti-inflammatory effects? Thromb Haemost 2017; 117(03):437-444

61 Esmon CT. Targeting factor Xa and thrombin: impact on coagulation and beyond. Thromb Haemost 2014;111(04): 625-633

62 Borissoff JI, Otten JJ, Heeneman S, et al. Genetic and pharmacological modifications of thrombin formation in apolipoprotein e-deficient mice determine atherosclerosis severity and atherothrombosis onset in a neutrophil-dependent manner. PLoS One 2013;8(02):e55784

63 Jeraj L, Jezovnik MK, Poredos P. Rivaroxaban versus warfarin in the prevention of post-thrombotic syndrome. Thromb Res 2017; $157: 46-48$ 